\title{
Platelet Aggregation: Prognostic Marker in Burn Survival
}

\author{
Muhammad Haisum Maqsood Bajwa, ${ }^{1}$ Sungeen Khan $^{2}$
}

Dear Sirs/Madams,

Burns are a global public health problem. They account for an estimated 265,000 deaths annually ${ }^{1}$. An accurate and objective tool for prognosis in patients with severe burns would be extremely valuable to medical professionals. Allowing doctors to forecast the probable outcome of burns and the prospects of recovery as indicated by the nature of the burns and the symptoms of the case. ${ }^{2}$

Traditionally, prediction of mortality among these patients has been made by employing Total Burn Surface Area and the patient's age. ${ }^{3,4}$ The relationship of platelet aggregation with mortality in burn victims has not been studied extensively and demands further exploration.

The degree of platelet aggregation can be measured inadvertently by the platelet count which will fall drastically if there is excessive platelet aggregation. A significantly lower platelet count has been found in non survivors of burn injury than in survivors and in one of these studies a consistently low platelet count

Date of Submission 14-11-2015

Date of Revision Received 19-1-2016

Date of Acceptance for Publication 29-1-2016

Conflict of Interest: None

Funding Source: None

Bajwa M.H.M. ${ }^{1}$

Department of Community Medicine, King Edward Medical University, Lahore

Khan S. ${ }^{2}$

Department of Community Medicine, King Edward Medical University, Lahore $\left[<1,00,000\right.$ per $\left.\mathrm{mm}^{3}\right]$ for 4 days uniformly resulted in death and platelet count decline has been found to precede other signs of sepsis among hospitalized burn victims. ${ }^{5}$ Activation of coagulation in trauma and burn patients is a forceful innate defence mechanism which may be an evolutionary adaptation to trauma in order to prevent bleeding and death. In general, burn patients have normal coagulation parameters at admission but become hypercoagulable during recovery. However, those who are hypercoagulable at admission may have an increased risk of venous thromboembolism. The role of fibrinogen in platelet aggregation after burn has been studied but no mortality correlation has been investigated. Modulation of platelet function in burn patients using aspirin in antithrombotic dose [150 mg/ day] has been found to enhance wound healing. Platelet function may be an important factor in the pathogenesis of micro thrombosis in the burn wound site resulting in deepening of the burn wound. Elevated thrombopoeitin in plasma of burned patients suggests that activated platelets amplify the inflammatory reactions and favour insurgence of organ damage in these pathologic conditions.

In addition to this we would like to assess the quality of the platelets in different burn victims and explore its implication in the survival and recovery of aforementioned patients. The quality of platelets may be determined with the help of platelet aggregometer as shown in Figure 1.

This area is completely open to research. The role of platelet aggregation in prognosis and survival of burn patients is yet to be investigated. We hypothesize that increased platelet aggregation will be associated with an increased mortality rate among burn victims and that a decrease in the quality of platelets is correlated with healing and survival. The data shall be collected by team members via a data collection tool from 60 burn victims admitted to Mayo Hospital (Lahore, 




Figure 1:

Pakistan); all of whom will be adults having a total body burn percentage in the range of $30-40 \%$. Persons with burns greater or lower than this range will be excluded. This decision has been undertaken as to minimize the effect that extent of trauma will have on outcome of this study.

The result of this study can be useful in establishing a new and more accurate prognostic tool for burn victims which can be done by correlating the relationship between survival v/s Platelet Aggregation against survival v/s the Revised Baux Scale. In addition to this, the results of this research can be used in designing new treatments for burn victims in relation to their extent of clotting and time since burn.

\section{Acknowledgements}

We would like to thank Dr Mustehsan Bashir Department of Plastic Surgery, King Edward Medical University, Lahore and Dr Ambreen Hameed, Department of Pathology, King Edward Medical University.

\section{Conflict of Interest}

None declared.

\section{References}

1. Who.int. WHO | Burns [Internet]. 2016 [cited 14 February 2016]. Available from:

http://www.who.int/violence_injury_prevention/other_i njury/burns/en/

2. Medical Dictionary | Prognosis [Internet]. [Cited on 18 March 2016]. Available from: http://medical-dictionary. thefreedictionary.com/Prognosis

3. Macedo J, Santos J. Predictive factors of mortality in burn patients. Revista do Instituto de Medicina Tropical de São Paulo. 2007; 49 (6): 365-370.

4. Osler T, Glance L, Hosmer D. Simplified Estimates of the Probability of Death After Burn Injuries: Extending and Updating the Baux Score. The Journal of Trauma: Injury, Infection, and Critical Care, 2010; 68 (3): 690697.

5. Housinger T. The Relationship between Platelet Count, Sepsis, and Survival in Paediatric Burn Patients. Arch Surg. 1993; 128 (1): 65. 